\title{
PREVALENCE OF MODIFIABLE RISK FACTORS AMONG STROKE PATIENTS IN A TERTIARY CARE HOSPITAL IN DHAKA
}

\author{
MD.BADIUZZAMAN ${ }^{1}$, FAZLE RABBI MOHAMMED ${ }^{2}$,FAZLE RABBI CHOWDHURY ${ }^{2}$, MD. SHAFIQUL BARI ${ }^{3}$, \\ MD. BILLAL ALAM ${ }^{4}$,H.A.M. NAZMUL AHASAN ${ }^{5}$
}

\begin{abstract}
:
Background: An event of stroke can be ignited by a number of risk factors, some of which are nonmodifiable and some are modifiable. Hypertension, diabetes mellitus, different heart diseases, hyperlipidemia and smoking belong to the latter group and their prevalence shows immense diversity worldwide. In this study we tried to identify the most prevalent risk factor for stroke. Categorization of various types of stroke and history of noncompliance in medication has also been evaluated.

Methods: This direct observational study was carried out on 400 patients of stroke admitted in different medicine units of Dhaka Medical College Hospital from July to December, 2007. Only patients having clinical diagnosis of stroke, confirmed by CT scan or MRI, were recruited. Patients were grouped into ischemic or hemorrhagic stroke or sub arachnoid hemorrhage.

Results: The male, female ratio was found 1.2:1. In the study cerebral infraction, intra cerebral hemorrhages (ICH) and sub arachnoid hemorrhage (SAH) were found in $56.25 \%$, 38.25\% and $5.5 \%$ patients respectively. In case of Ischemic stroke and ICH males were predominantly affected but in case of SAH females were predominant. Among patients, hypertension was found in $58.62 \%$ followed by smoking (53.79\%), lipid disorder (48.01\%), heart diseases (25.75\%), diabetes mellitus (20.01\%), and previous history of stroke (10.61\%). 40\% patients were on irregular use of antihypertensive drug and it was $17.5 \%$ in case of anti diabetic drugs. Major groups (42.44\%) of patients have two modifiable risk factors.

Conclusion: Stroke is better to prevent than to cure. Identification of risk factors is the only way to achieve this goal. Implementation of screening programme in community to identify risk factors and educate people about primary prevention should be initiated in this regard. This way, we can reduce morbidity and mortality among stroke patients and alleviate the burden of stroke.
\end{abstract}

\section{Introduction:}

Stroke is the third most common cause of death after coronary heart disease and all cancer deaths. According to the World Health Organization (WHO), 15 million people suffer stroke worldwide each year. Of these, 5 million die and another 5 million are permanently disabled. ${ }^{1}$ There is no doubt that stroke is not only the leading cause of long-term disability in Bangladesh but also it generates an enormous economic burden for individuals, families and communities. Sufficient data are not available as no large study is done yet in Bangladesh. Some studies in Pakistan revealed that hypertension was the most common modifiable risk factor (43-66\%). Other important modifiable risk factors include diabetes mellitus (27-42\%), dyslipidemia (19-30\%), smoking (11$43 \%)$, ischaemic heart disease (9-46\%), previous stroke or TIA (11-26\%). ${ }^{2}$ Direct and indirect costs for stroke in 2005 are estimated at $\$ 56.8$ billion in the United States. ${ }^{3}$ Prevention is the only way to lower this giant financial burden and the identification of modifiable risk factors is required in this context. In this study, prevalence of various modifiable risk factors among stroke patients in a tertiary care hospital has been identified.

1. Indoor Medical Officer, Department of Medicine, Dhaka Medical College Hospital, Dhaka.

2. Post-graduate Trainee, Department of Medicine, Dhaka Medical College Hospital, Dhaka.

3. Associate Professor, Department of Medicine, Sylhet MAG Osmani Medical College, Sylhet.

4. Associate Professor, Department of Medicine, Dhaka Medical College, Dhaka.

5. Professor, Department of Medicine, Dhaka Medical College, Dhaka.

Correspondence : Dr. Md.Badiuzzaman, Indoor Medical Officer, Department of Medicine, Dhaka Medical College Hospital, Dhaka. 


\section{Materials and Methods:}

This study was a direct observational study conducted among 400 stroke patients admitted in different Medicine units of Dhaka Medical College Hospital. The objective of the study was to assess the association of modifiable risk factors of stroke and to identify the most vulnerable risk group. Study period was from July to December, 2007. 400 respondents and their reliable attendants were interviewed by pre tested structured questionnaire.

Only patients having clinical manifestation of stroke persisting for more than 24 hours were included after taking informed written consent. CT scan or MRI was done in every case. Presences of hypertension (HTN), diabetes mellitus (DM), hyperlipidemia, smoking and different type of heart diseases including ishaemic heart disease (IHD), valvular heart disease (VHD), atril fibrillation (AF), infective endocarditis (IE) was evaluated. HTN was defined according to British Hypertension Society; diabetes fulfilled the diagnostic criteria described by WHO and hyperlipidemia defined by ATP III classification. Routine and different special investigations were also done when required. Transient elevation of blood pressure, which lowered down without treatment by 7 days after stroke, was excluded. Demographic variables were included. Patients were grouped in three forms of stroke including cerebral infarction, intracerebral hemorrhages (ICH) and sub arachnoid hemorrhage (SAH).

\section{Results:}

Out of 400 patients with stroke, 215 (53.75\%) were male and 185 (46.25\%) were female (M: $\mathrm{F}=1.2: 1)$

In our study cerebral infraction, ICH and $\mathrm{SAH}$ were found in 225 (56.25\%), 153 (38.25\%) and 22 (5.5\%) patients respectively. In case of Ischemic stroke and $\mathrm{ICH}$, males were predominantly affected but in case of SAH females were predominant. (Fig.-1)

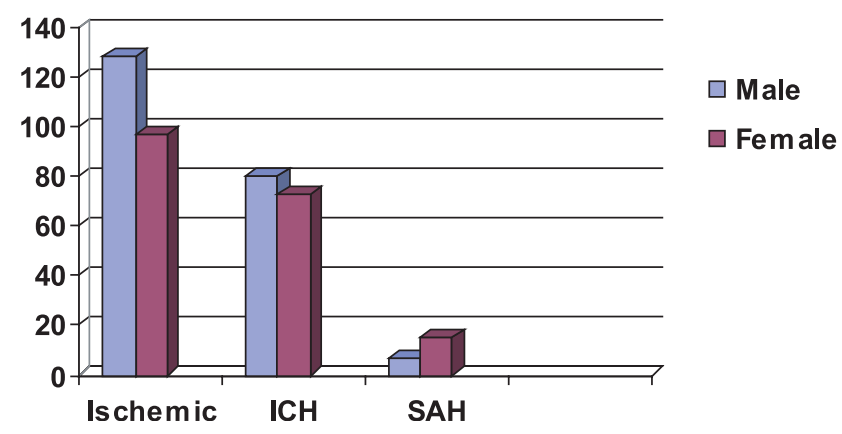

Fig.-1: Distribution of different types of stroke in different $\operatorname{sex}(n=400)$.
In our study, maximum patients (42.44\%) had at least two modifiable risk factors. $32.09 \%$ patients had one, $19.62 \%$ had three and only $1.32 \%$ had five risk factors respectively. $5.75 \%$ had no risk factors at all.

Out of 121 patients (32.09\%) who had at least one risk factor, 55 had hypertension, 40 had lipid disorders, 20 were smoker, 4 had diabetes mellitus, 2 had previous history of stroke. (Fig.-2)

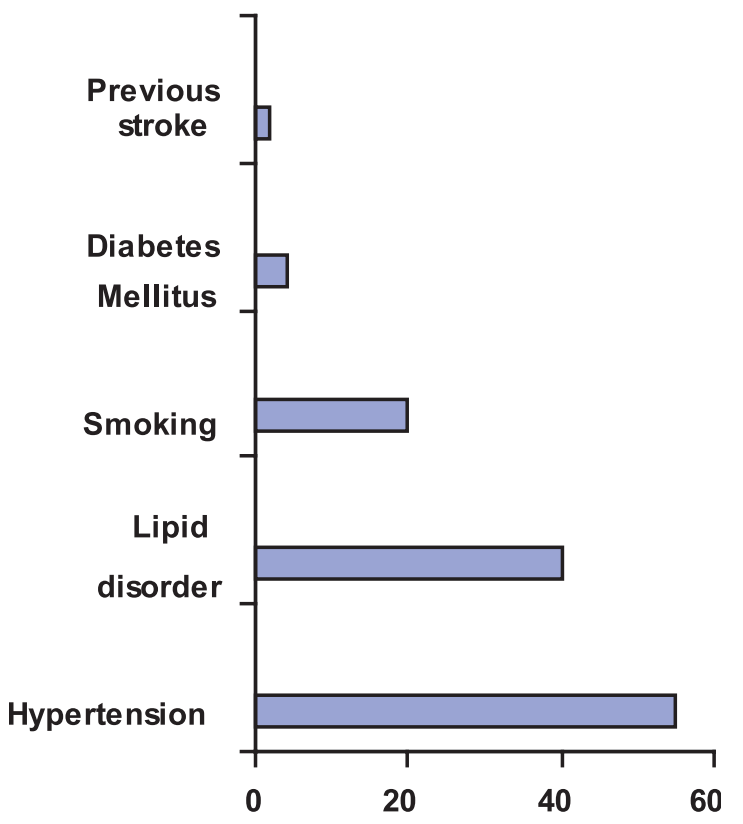

Fig.-2: Distribution of patients according to individual risk factors. $(n=121)$

We categorized patients having multiple risk factors. $58.62 \%$ patients had hypertension with other risk factors and this was followed by risk factors associated with smoker (53.9\%), lipid disorder (48.01\%), heart diseases (25.75\%), diabetes mellitus (20.01\%) and previous history of stroke (10.61\%).

Among the patients, $62 \%$ were previously diagnosed as hypertensive, $2.9 \%$ were diagnosed for the first time during stroke and $34.75 \%$ were normotensive. In this respect, no marked discrimination was present regarding gender. Among the known cases of hypertensive patients, $40.40 \%$ were on irregular use of antihypertensive drug with an insignificant male, female ratio (1.2:1). Out of 76 patients with diabetes mellitus (20.01\%), $20 \%$ were known cases and among them only $17.5 \%$ were on irregular use of anti diabetic drugs.

Among the patients (181) with lipid disorder, most of them had low high density lipoprotein (HDL) (42.67\%). 
$27.93 \%, 21.08 \%$ and $13.41 \%$ patients with lipid disorder had high cholesterol, low density lipoprotein (LDL) and triglycerides (TG) level respectively. 88.25\% patients with lipid disorder were undiagnosed previously.

Out of 115 smokers, only $1.5 \%$ was female and incidence of stroke was higher who had a history of 11 to 30 pack years of smoking.

Out of 102 patients (25.75\%), who had heart diseases, $21.25 \%$ had IHD of which $13.5 \%$ were detected for the first time. IHD was followed by VHD (4\%), AF (0.25\%) and IE $(0.25 \%)$.

Out of total cases, 40 patients had previous history of stroke with a male predominance (M:F : 2.73:1).

\section{Discussion:}

Stroke is a clinical syndrome characterized by rapidly developing symptoms and/or signs of focal and at times global (for patients in coma) loss of cerebral functions, with symptoms lasting more than 24 hours or leading to death with no apparent cause other than that of vascular origin. ${ }^{4}$ Out of 400 patients, male, female ratio was 1.2:1 and this results correlates with the study done by Rahman M H in 1997 in Sir Salimullah Medical College and Mitford Hospital from July to December 1996 and it was 1.8:1. ${ }^{5}$

In our study, cerebral infraction, ICH and SAH were found in $56.25 \%, 38.25 \%$ and $5.5 \%$ patients respectively. Our study differs largely from most of the western studies where cerebral infarction, ICH and SAH comprises $85 \%, 10 \%$ and $5 \%$ cases of stroke respectively. ${ }^{6,7}$ But a study which was done by Khan M M Z et al in Bangladesh showed a similar type of outcome. ${ }^{8}$ Long standing hypertension results in atherosclerosis which increases the incidence of cerebral infarction. But sudden rise of blood pressure is typically associated with ICH. Most of the people in western world get good health services and they are well educated to take antihypertensive drugs regularly. As because long standing HTN causes cerebral infarction, it is commoner in western world than ICH. In Bangladesh, we fail to provide adequate health care services and more over poor people fail to take antihypertensive regularly. As a result, fluctuation of blood pressure is commoner in this country which results ICH. In our study, $43.75 \%$ patients had hemorrhagic stroke and $40.40 \%$ were on irregular use of antihypertensive drug. Among patients, hypertension was found in $58.62 \%$ followed by smoking (53.79\%), lipid disorder (48.01\%), heart diseases (25.75\%), diabetes mellitus (20.01\%), and previous history of stroke (10.61\%). HTN is the most important risk factor of stroke. This result correlates with that of Khan M M Z et al, Khan J et al or Vohra's report. ${ }^{8-10}$

The incidence of stroke in smokers was found in 53.79 $\%$ cases and this differs from Khan M M Z's study but co relates with Khan J. ${ }^{8,9}$ In relation to pack year of smoking, the incidence was higher among those who smokes 11-30 pack year. In this study, dyslipidemia was detected as the 3rd leading factor of stroke. Total cholesterol was found high in $27.93 \%$ cases which is similar to that reported by Khan S N et.al. ${ }^{11}$ Different types of heart disease found in $25.50 \%$ cases which is similar to that reported by Khan M M Z (25\%) and Khan S N (26\%). ${ }^{8,11}$ In our study, $89.22 \%$ patients with heart disease had IHD. DM was another important risk factor in association with stroke. In this study the incidence of DM was $20.01 \%$ which is almost similar to that reported by Khan M M Z (20\%) but differs from that reported by Khan J (33\%) or Khan S N (41\%). 8,9,11 Out of known cases of DM only $17.5 \%$ were on irregular medication. In most study, $\mathrm{DM}$ is the second commonest risk factor of stroke. But in our country, the incidence of DM in relation to stroke is much lower than that of western country. $10.61 \%$ patients had previous history of stroke and the incidence was higher among ischemic stroke.

This small hospital based study may not reflect the exact scenario of stroke in the community. But several risk factors like HTN, DM, Smoking, Dyslipidemia, IHD are observed here. Stroke is better to prevent than to cure. Identification of risk factors is the only way to achieve this goal. Implementation of screening programme in community to identify risk factors and educate people about primary prevention should be initiated in this regard. This way, we can reduce morbidity and mortality among stroke patients and alleviate the burden of stroke.

\section{References:}

1. World Health Report - 2007. World Health Organization. International Cardiovascular Disease Statistics (2007 Update).In: American Heart Association. Available at: http://www.strokecenter. org/pat/stats.htm.

2. Bhojo A. Khealani, Bilal Hameed, Uzma U. Mapari. Stroke in Pakistan. J Pak Med Assoc 2008; 58 (7) : 400-403. 
3. Heart Disease and Stroke Statistics - 2005 Update. In: American Heart Association. Available at: http:/ /www.americanheart.org/downloadable/heart/ 1105390918119HDSStats2005Update.pdf.

4. Davenport R, Denis M. Neurological emergencies: Acute stroke. J Neurol Neurosurg Psychiatry 2000; 68:277-88.

5. Rahman MH. Study of risk factors and their correlation will 100 hospitalized patients of stroke. Dhaka: SSMC and Mitford Hospital, 1996.

6. Broderick JP, Brott T, Tomsick T, Miller R, Huster G. Intracerebral hemorrhage more than twice as common as subarachnoid hemorrhage. J Neurosurg. 1993;78: 188-191.

7. Smith WS, Johnston S C, Easton J D. Cerebrovascular Diseases. In: Fauci AS, Braunwald
E, Kasper DL, et al.17th edition. Harrison's Internal Medicine .New York: The McGraw-Hill Companies, 2008: Ch 364.

8. Khan MMZA, Arif SM, Parveen S et. al.Unconscious patients and stroke - a study of 50 cases. journal of Medicine 2005; 6: 5-9.

9. Khan J, Rehman AU, A Ali Shah, Jielani A. Frequency of hypertension in stroke patients presenting at Ayub Teaching Hospital. J Ayub Med Coll Abbottabad 2006;18: 59-61.

10. Vohra EA, Ahmed WU, Ali M. Etiology and prognostic factors of patients admitted for stroke.J Pak Med Assoc 2000;50(7);234-6.

11. Khan SN, Vohra EA. Risk factors for stroke: A hospital based study. Pak J Med Sci 2007; 23: 17-22. 